\title{
DNA damage response protects against progressive kidney disease
}

\author{
Bruce A. Molitoris
}

Department of Medicine, Indiana University School of Medicine, Indianapolis, Indiana, USA.

\begin{abstract}
The pathophysiology of cellular injury and repair has been extensively studied in acute kidney injury (AKI) for more than 70 years. Although a great deal of knowledge has been generated, a debate over the importance of repairing damaged cells versus replacing them by proliferation remains. In this issue of the $J C l$, Kishi et al. demonstrate that following kidney epithelial cell injury, DNA repair, rather than cell proliferation, plays the central role in recovery and longevity by minimizing apoptosis, $\mathrm{G}_{2} / \mathrm{M}$ cell-cycle arrest, and subsequent fibrosis. This has important therapeutic implications and highlights the need for more sensitive techniques to evaluate functional, structural, and molecular recovery following injury.
\end{abstract}

\section{The DNA damage response in kidney injury}

Cellular DNA damage can be caused by exogenous insults, such as drugs and UV light, or endogenous stressors such as ROS. Damage includes single- and double-strand breaks, base loss, and mismatched base pairs (1). A sensitive and complex DNA damage response (DDR) has evolved to detect these lesions, delay cell-cycle progression, and repair the damage. Two major sensors of DNA damage include ataxia telangiectasia mutated (ATM) and ATM and Rad-3-related (ATR). ATR senses single-strand DNA breaks and DNA cross-linking and phosphorylates and activates the checkpoint protein Chk1. In turn, Chk1 regulates cyclin-dependent kinase 1 (CDK1) and CDK2, resulting in $\mathrm{G}_{1} / \mathrm{S}, \mathrm{S}$, and $\mathrm{G}_{2} / \mathrm{M}$ arrest to allow for DNA replication.

However, if DNA repair is incomplete, cell dysfunction, cell-cycle arrest, senescence, and, eventually, cell death will follow. In the context of acute kidney injury (AKI), cell-cycle arrest been shown to lead to enhanced cytokine secretion and fibrosis, which drive chronic kidney disease (CKD) (2). Pioneering work from the Dong lab- oratory previously demonstrated that the DDR is activated in cisplatin and ischemiainduced AKI (3-5), highlighting the pathway as a therapeutic target to protect against kidney damage. However, the potential clinical importance of specific components of the pathway and the compartments of the kidney in which they act remained unknown.

In this issue, Kishi and colleagues (6) hypothesized that the DDR plays a critical role in renal proximal tubule (PT) cells, such that loss of the pathway would impair adaptive cellular DNA repair and hasten fibrotic changes. To test their hypothesis, they used an extensive series of models, including tissue from patients with $\mathrm{CKD}$ and from mice with PT-specific deletion of ATR, human kidney organoids, and cultured PT cells. These studies offer new insights into the pathophysiology of AKI cellular recovery and progression to CKD.

\section{From bedside to bench}

One never knows the actual order in which studies were performed, but starting with human data is always fine, as it gives increased assurance that the studies have clinical relevance. The authors found that

Related Article: p. 4797

Conflict of interest: The author has declared that no conflict of interest exists.

biopsies from patients with CKD showed more extensive evidence of ongoing cellular injury (KIM-1 positive) and DNA damage $(\gamma \mathrm{H} 2 \mathrm{AX}$ positive) than did biopsies from patients with minimal change disease (MCD) (6). Notably, the level of ATR was increased in KIM-1-stained cells, although the correlation was biased by one point and should be interpreted with caution. The authors also concluded that the degree of DNA damage was inversely correlated with the estimated glomerular filtration rate (eGFR), although this result may be skewed by the inclusion of patients with MCD as the control.

The authors next used a mouse model with PT cell-specific deletion of ATR (6). In the cisplatin-induced AKI model, ATR-deficient mice displayed increased mortality and worsened functional impairment, tubular injury scores, and KIM-1 staining. In these mice, the injury was greater in the outer medulla of the kidney than in the cortex. They also had increased $\gamma \mathrm{H} 2 \mathrm{AX}$ foci, a likely indication of increased double-strand DNA breaks. Subsequent studies in cultured cells and human kidney organoids, using the known ATR inhibitor VE-821, extended the observations made in the mouse model. Most important, the authors confirmed the increase in $\mathrm{G}_{2} / \mathrm{M}$ cell-cycle arrest and showed that it was dependent on p53. The ability to replicate many of the in vivo findings in organoids and cell culture studies is remarkable and will allow these investigators to move rapidly to understand this complex repair process and screen potential therapeutics.

As mentioned above, previous work from this group demonstrated an association between $\mathrm{G}_{2} / \mathrm{M}$ cell-cycle arrest and cytokine secretion and fibrosis (2). Consistent with this model, ATR-deficient mice subjected to ischemia-reperfusion kidney injury showed increased cell loss, delayed functional recovery, upregulation of profibrotic genes, enhanced extracellular matrix collagen deposition, and PT cell senescence-associated $\beta$-gal staining (6). 


\section{Cell repair versus cell proliferation}

The debate regarding the relative contribution of cell repair versus cell proliferation to recovery from AKI has a long history with many chapters. Early morphological and functional studies clearly showed that the rate and extent of cellular repair and recovery is dependent on the extent of ischemic injury $(7,8)$. Recovery of PT cells after ischemic injury is especially rapid in PT segments 1 and 2, but far less so in segment 3 , where the return of blood flow is less reliable, resulting in a patchy pattern of continued injury, apoptosis, and necrosis (9). This remarkable morphological recovery of the $\mathrm{PT}$, the main site of cellular injury, led to a false conception that complete normalization after injury was possible. An understanding of this process was further complicated by the fact that the kidney can increase functional capacity above baseline function (renal functional reserve $[\mathrm{RFR}]$ ), which allows serum creatinine to return to baseline after injury even though total kidney function (baseline plus RFR) has been reduced $(10,11)$.

Studies using BrdU, a thymidine substitute, identified DNA synthesis as an early event in PT cells in many different AKI models. This initiated a robust search for stem cells that were predicted to be responsible for repopulating the basement membrane. However, BrdU is also incorporated into DNA during repair and thus labels both proliferating and nonproliferating cells undergoing DNA repair. Subsequent lineage-tracing studies have shown that injured PT cells, rather than a fixed tubular progenitor, undergo dedifferentiation and clonal proliferation to reconstitute the areas of severe cell injury (12). However, in both rodents and patient samples, it appears that this dedifferentiation and proliferation occur primarily in the PT segment 3 cells within the cortical-medullary or outer stripe of the medulla, and not in the outer cortex (9). This is due to vascular reperfusion abnormalities within this region. Therefore, the majority of cortical cells undergo DNA repair and not dedifferentiation and proliferation following injury. However, if the DNA repair is incomplete or faulty, then the scenario described by Kishi et al. (6) could play a major role in subsequent maladaptive repair including reduced functional recovery, apoptosis, $\mathrm{G}_{2} / \mathrm{M}$ arrest, cytokine production, and fibrosis.

\section{Looking forward}

A better understanding of DNA repair, including identification of the factors limiting injury and facilitating recovery, may lead to therapeutic approaches that can be used to prevent DNA damage or improve the repair response. In this regard, the authors also point out the potential for harm from small-molecule inhibitors of ATR presently being developed as adjuvants for chemotherapy regimens. This may be especially important in patients with CKD with ongoing chronic ischemia, as the authors showed that DNA repair was continuous in this context. One last note of caution: a recent study showed that ATR activation in nonreplicating cells is regulated by the XPB subunit of transcription factor IIH and could lead to cell death in nonreplicating cells (13). Consistent with this finding, ATR-mediated DNA damage signaling resulted in kidney tubule epithelial cell death in models of severe AKI $(5,6)$. Therefore, efforts to manipulate the DNA repair pathway therapeutically should be carefully considered.

\section{Acknowledgments}

Bruce A. Molitoris is supported by $\mathrm{NIH}$ grants RO1DK091623 and P30DK079312.

Address correspondence to: Bruce A. Molitoris, Research-2, Room 266, 950 West Walnut Street, Indianapolis, Indiana
46202, USA. Phone: 317.274.1251; Email: bmolitor@iu.edu.

1. Jackson SP, Bartek J. The DNA-damage response in human biology and disease. Nature. 2009;461(7267):1071-1078.

2. Yang L, Besschetnova TY, Brooks CR, Shah JV, Bonventre JV. Epithelial cell cycle arrest in G2/M mediates kidney fibrosis after injury. Nat Med. 2010;16(5):535-543.

3. Pabla N, Bhatt K, Dong Z. Checkpoint kinase 1 (Chk1)-short is a splice variant and endogenous inhibitor of Chk1 that regulates cell cycle and DNA damage checkpoints. Proc Natl Acad Sci U S A. 2012;109(1):197-202.

4. Pabla N, Huang S, Mi QS, Daniel R, Dong Z. ATR-Chk2 signaling in p53 activation and DNA damage response during cisplatin-induced apoptosis. J Biol Chem . 2008;283(10):6572-6583.

5. Pabla N, Ma Z, McIlhatton MA, Fishel R, Dong Z. hMSH2 recruits ATR to DNA damage sites for activation during DNA damage-induced apoptosis. J Biol Chem. 2011;286(12):10411-10418.

6. Kishi S, et al. Proximal tubule ATR regulates DNA repair to prevent maladaptive renal injury responses. J Clin Invest. 2019;129(11):4797-4816.

7. Glaumann B, Glaumann H, Berezesky IK, Trump BF. Studies on the pathogenesis of ischemic cell injury. II. Morphological changes of the pars convoluta (P1 and P2) of the proximal tubule of the rat kidney made ischemic in vivo. Virchows Arch B Cell Pathol. 1975;19(4):281-302.

8. Glaumann B, Trump BF. Studies on the pathogenesis of ischemic cell injury. III. Morphological changes of the proximal pars recta tubules (P3) of the rat kidney made ischemic in vivo. Virchows Arch B Cell Pathol. 1975;19(4):303-323.

9. Takasu O, et al. Mechanisms of cardiac and renal dysfunction in patients dying of sepsis. Am J Respir Crit Care Med. 2013;187(5):509-517.

10. Molitoris BA. Rethinking CKD evaluation: should we be quantifying basal or stimulated GFR to maximize precision and sensitivity? Am J Kidney Dis. 2017;69(5):675-683.

11. Palsson R, Waikar SS. Renal functional reserve revisited. Adv Chronic Kidney Dis. 2018;25(3):e1-e8.

12. Chang-Panesso M, Humphreys BD. Cellular plasticity in kidney injury and repair. Nat Rev Nephrol. 2017;13(1):39-46.

13. Kemp MG. DNA damage-induced ATM- and Rad-3-related (ATR) kinase activation in non-replicating cells is regulated by the XPB subunit of transcription factor IIH (TFIIH). J Biol Chem. 2017;292(30):12424-12435. 